\title{
Avaliação da Perda Auditiva em Doentes com Tuberculose Multirresistente
}

\author{
Evaluation of Hearing in Patients with Multiresistant Tuberculosis
}

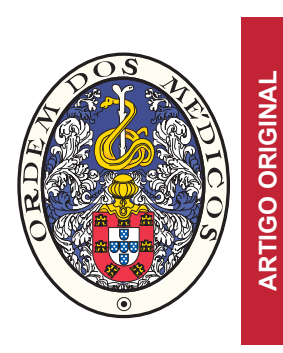

\author{
Leandro RIBEIRO' ${ }^{1}$, Claudia SOUSA², Aida SOUSA ${ }^{1}$, Catarina FERREIRA ${ }^{3}$, Raquel DUARTE ${ }^{4}$, \\ António FARIA E ALMEIDA ${ }^{1}$, Artur CONDÉ1 \\ Acta Med Port 2015 Jan-Feb;28(1):87-91
}

\section{RESUMO}

Introdução: A tuberculose multirresistente e a extensivamente resistente constituem atualmente a principal ameaça ao controlo da tuberculose a nível mundial. O seu tratamento exige o uso de um esquema terapêutico com fármacos de segunda linha por um período mínimo de 20 meses, onde se incluem aminoglicosídeos durante um período de cerca de oito meses. Estes estão associados a alterações cocleovestibulares permanentes.

Material e Métodos: Estudo transversal que pretende avaliar a função auditiva em indivíduos que terminaram tratamento por tuberculose multirresistente (com esquema que incluia aminoglicosídeos) no Centro de Referência Regional para a Tuberculose Multirresistente da Sub-Região de Saúde do Norte entre os anos de 2009 e 2012. Os doentes que preencheram os critérios de inclusão foram convocados para a realização de: exame objetivo otorrinolaringológico, audiograma tonal e vocal, otoemissões acústicas por produtos de distorção nas frequências de $2000-5000 \mathrm{~Hz}$ e timpanograma.

Resultados: Entre 2009 e 2012 foram referenciados 27 casos para o Centro de Referência para a tuberculose multirresistente da Região Norte, tendo-se verificado cinco óbitos. Dos 22 indivíduos convocados, compareceram 11. Estes possuíam idades compreendidas entre os 31 e os 59 anos ( $41 \pm 10)$. A amicacina foi usada em $80 \%$ e a capreomicina nos restantes. Seis $(60 \%)$ dos utentes estudados apresentaram surdez neurossensorial. As otoemissões acústicas foram negativas em todos os doentes com hipoacúsia neurossensorial, o que confirma a sua origem coclear.

Discussão/ Conclusão: Os resultados deste estudo sugerem a necessidade de existir uma monitorização audiológica mais frequente nos indivíduos com tuberculose multirresistente tratados com aminoglicosídeos permitindo detectar mais precocemente a perda auditiva.

Palavras-chave: Aminoglicosídeos; Perda Auditiva; Tuberculose Multirresistente.

\section{ABSTRACT}

Introduction: Multidrug-resistant tuberculosis and extensively drug-resistant tuberculosis constitute a major threat to worldwide tuberculosis control. The treatment demands the use of a therapeutic regimen in second-line drugs for a minimum period of 20 months, which includes aminoglycosides over a period of about eight months. These are associated with permanent cochleovestibular changes. Material and Methods: This cross-sectional study aims to evaluate the auditory function in individuals who completed treatment for multidrug-resistant tuberculosis (with scheme that included aminoglycosides) at the Center of Regional Reference for multidrugresistant tuberculosis located in the North of Portugal, between the years 2009 and 2012. Patients who met the inclusion criteria were submitted to tonal and vocal audiograms, otoacoustic emissions at 2000-5000 Hz frequencies, and a tympanogram.

Results: Between 2009 and 2012, 27 patients were referred to the Centre of Regional Reference for multidrug-resistant tuberculosis, in the North Region of Portugal, resulting in five deaths. Of the 22 individuals invited, 11 attended. These were aged between 31 and 59 years $(41 \pm 10)$. Amikacin was used in $80 \%$ of patients. Six patients $(60 \%)$ had sensorineural hearing loss.

Discussion/ Conclusion: The results of this study suggest the need for a more frequent audiological monitoring in patients with multidrug-resistant tuberculosis treated with aminoglycosides allowing early detect hearing loss.

Keywords: Aminoglycosides; Hearing Loss/ chemically induced; Tuberculosis, Multidrug-Resistant.

\section{INTRODUÇÃO}

A tuberculose multirresistente (TB-MDR), definida como a ocorrência de resistência simultânea à isoniazida e à rifampicina, e a tuberculose extensivamente resistente (TB-XDR), considerada como TB-MDR com resistência associada às quinolonas $e$ a, pelo menos, um dos seguintes - amicacina, canamicina e capreomicina - em estirpes de Mycobacterium Tuberculosis, ${ }^{1-4}$ constitui atualmente, aliado à co-infeção por $\mathrm{VIH}$, a principal ameaça ao controlo da tuberculose a nível mundial. ${ }^{5,6} \mathrm{O}$ seu tratamento exige o uso de um esquema terapêutico alternativo com fármacos de segunda linha por um período mínimo de 20 meses $^{3}$, onde se incluem aminoglicosídeos durante um período de cerca de 8 meses. Estes estão associados a danos cocleovestibulares permanentes. . $^{1-10}$

A Direção Geral da Saúde (DGS) criou em Junho de 2007 o Centro de Referência Nacional para a TB-MDR através da publicação da Circular Informativa no 14/DT de 05 de Junho de 2007. Nesse documento propôs, de igual forma, a criação de Centros de Referência Regionais para a TB-MDR em cada uma das cinco Regiões de Saúde e na

1. Serviço de Otorrinolaringologia. Centro Hospitalar de Vila Nova de Gaia/Espinho. Vila Nova de Gaia. Portugal.

2. Departamento de Audiologia. Escola Superior de Tecnologias da Saúde. Porto. Portugal.

3. Serviço de Pneumologia. Centro Hospitalar e Universitário de Coimbra. Coimbra. Portugal.

4. Centro de Referência Regional para a Tuberculose Multisseristente da Região de Saúde do Norte. Vila Nova de Gaia. Portugal.

Recebido: 01 de Setembro de 2014 - Aceite: 23 de Dezembro de 2014 | Copyright @ Ordem dos Médicos 2015 
Região Autónoma da Madeira. No âmbito do Programa de Luta Contra a Tuberculose da Administração Regional de Saúde do Norte 2008-2010, e dando cumprimento à proposta da DGS, é criado o Centro de Referência Regional para a TB-MDR da Região de Saúde do Norte (CRRTB-MDR-Norte), o qual é responsável pelo acompanhamento e apoio ao tratamento de todos os doentes portadores de TB-MDR da respetiva região.

Faz parte da rotina do CRRTB-MDR-Norte que os doentes sejam orientados para consultas de Otorrinolaringologia da sua área de residência no decorrer do tratamento.

\section{MATERIAL E MÉTODOS}

Este estudo de corte transversal e de natureza descritiva tem como objetivo avaliar a função auditiva em indivíduos que terminaram tratamento por TB-MDR/TB-XDR (com esquema que incluia aminoglicosídeos) no CRRTB-MDR-Norte entre os anos de 2009 e 2012. Foram excluídos os doentes com surdez congénita ou queixas cocleares prévias ao tratamento, bem como aqueles que apresentaram surdez de condução ou alterações otoscópicas na presente avaliação.

Os doentes que preencheram os critérios de inclusão foram convocados para realização de (1) anamnese e exame otorrinolaringológico, (2) audiograma tonal (AT) e vocal (AV), (3) AT de elevadas frequências (8-16 KHz), (4) otoemissões acústicas (OEA) automáticas por produtos de dis- torção nas frequências 2000-5000 Hz e (5) timpanograma.

$\mathrm{Na}$ anamnese foram inquiridas a presença de queixas cocleares, presentes ou prévias ao tratamento da tuberculose, a história ocupacional e a exposição a ruído. Foi igualmente determinado o tipo de tuberculose (pulmonar, ganglionar ou disseminada), o fármaco ototóxico utilizado, bem como o respetivo tempo de tratamento.

No audiograma tonal foram testadas as vias aérea e óssea, tendo-se calculado o Pure-tone average (PTA) (média dos limiares auditivos nas frequências $500-4000 \mathrm{~Hz}$ ). Foi definido o limiar de $25 \mathrm{~dB} \mathrm{HL}$, acima do qual os doentes foram considerados como portadores de perda auditiva. Esta foi classificada em:

I - surdez ligeira se PTA entre 26-40 dB HL;

II - surdez moderada se PTA 41-70 dB HL;

III - surdez severa se PTA 71-90 dB HL;

IV - surdez profunda se PTA $\geq 91 \mathrm{~dB} H \mathrm{HL}$.

No audiograma vocal foi calculado o Speech Reception Threshold (SRT). As OEA foram classificadas como presentes ou ausentes, e o timpanograma em tipos A, B ou C.

\section{RESULTADOS}

No período de tempo compreendido entre os anos de 2009 e 2012 foram referenciados para o CRRTB-MDR-Norte 27 doentes com TB-MDR ou TB-XDR, tendo-se verificado 5 óbitos (18,5\%) em doentes com co-morbilidades (Tabela 1).

Tabela $1 \mathrm{~A}$ - Dados da avaliação auditiva em doentes com TB-MDR tratados no CRRTB-MDR-Norte (continua)

\begin{tabular}{|c|c|c|c|c|c|c|c|}
\hline \multirow[t]{2}{*}{ Caso } & \multirow[b]{2}{*}{$\begin{array}{l}\text { Idade } \\
\text { (anos) }\end{array}$} & \multirow[b]{2}{*}{ Lado } & \multicolumn{5}{|c|}{ AVALIAÇÃO AUDITIVA } \\
\hline & & & Tipo de TB & $\begin{array}{l}\text { Fármaco } \\
\text { ototóxico }\end{array}$ & $\begin{array}{c}\text { Duração de } \\
\text { tratamento } \\
\text { (meses) }\end{array}$ & $\begin{array}{c}\text { Fim do } \\
\text { tratamento } \\
\text { (meses) }\end{array}$ & $\begin{array}{c}\text { Timpanograma } \\
\text { (tipo) }\end{array}$ \\
\hline \multirow{2}{*}{1} & \multirow{2}{*}{31} & Direito & \multirow{2}{*}{$P^{1}$} & \multirow{2}{*}{ Amicacina } & \multirow{2}{*}{4} & \multirow{2}{*}{31} & A \\
\hline & & Esquerdo & & & & & A \\
\hline \multirow{2}{*}{2} & \multirow{2}{*}{44} & Direito & \multirow{2}{*}{$\mathrm{P}^{1}$} & \multirow{2}{*}{ Amicacina } & \multirow{2}{*}{6} & \multirow{2}{*}{36} & A \\
\hline & & Esquerdo & & & & & $A$ \\
\hline \multirow{2}{*}{3} & \multirow{2}{*}{59} & Direito & \multirow{2}{*}{$P^{1}$} & \multirow{2}{*}{ Amicacina } & \multirow{2}{*}{6} & \multirow{2}{*}{22} & A \\
\hline & & Esquerdo & & & & & A \\
\hline \multirow{2}{*}{4} & \multirow{2}{*}{36} & Direito & \multirow{2}{*}{$\mathrm{P}^{1}$} & \multirow{2}{*}{ Amicacina } & \multirow{2}{*}{5} & \multirow{2}{*}{24} & A \\
\hline & & Esquerdo & & & & & A \\
\hline \multirow{2}{*}{5} & \multirow{2}{*}{41} & Direito & \multirow{2}{*}{$\mathrm{D}^{2}$} & \multirow{2}{*}{ Capreomicina } & \multirow{2}{*}{8} & \multirow{2}{*}{4} & A \\
\hline & & Esquerdo & & & & & A \\
\hline 6 & 31 & Direito & $P_{1}$ & Amicasing & 8 & & A \\
\hline 0 & 31 & Esquerdo & $P$ & Amıcacına & 8 & 23 & A \\
\hline 7 & 52 & Direito & $D_{1}$ & Amisosing & 7 & 19 & A \\
\hline 7 & 53 & Esquerdo & $P$ & Amıcacına & 7 & 19 & A \\
\hline 0 & ? & Direito & 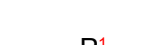 & A min & 0 & 11 & $A$ \\
\hline 0 & 32 & Esquerdo & $r$ & Hinluacila & 0 & II & A \\
\hline & 10 & Direito & & & 0 & & $A$ \\
\hline 9 & 49 & Esquerdo & $P$ & Capreomıcına & 8 & 30 & A \\
\hline 10 & 31 & Direito & $a^{3}$ & Amicosing & 8 & 1 & A \\
\hline 10 & 34 & Esquerdo & $\checkmark$ & Amicacina & 0 & 1 & A \\
\hline
\end{tabular}


Compareceram neste estudo 11 dos 22 indivíduos convocados, 7 do sexo feminino e 4 do sexo masculino, o que reflete uma taxa de resposta de $50 \%$. Um indivíduo foi excluído do estudo por apresentar surdez congénita. A TB-MDR foi a forma de TB encontrada em todos os indivíduos avaliados. Nenhum dos doentes apresentava queixas cocleovestibulares prévias ao tratamento instituído. Da mesma forma, nenhum apresentou alterações otoscópicas.

A média de idades, dos dez doentes avaliados, foi de 41 ( \pm 10$)$ anos e o tempo de tratamento com fármacos ototóxicos variou entre os 4 e os 8 meses $(6,6 \pm 1,4)$. A interrupção precoce esteve associada, frequentemente, com o aparecimento de toxicidade renal ou auditiva.

A amicacina foi o fármaco mais utilizado (80\%).

A incidência da perda auditiva neste estudo foi de $65 \%$, o que corresponde a 13/20 ouvidos: 2/20 ouvidos com surdez NS ligeira (10\%), 7/20 ouvidos com surdez NS moderada (35\%), e 2/20 ouvidos com surdez NS severa e profunda (10\%), respetivamente.

Na Fig. 1 estão representadas as médias dos limiares auditivos em cada uma das frequências testadas (12516.000 Hz).

\section{DISCUSSÃO/CONCLUSÃO}

De acordo com o nosso conhecimento, este é o primeiro trabalho realizado em Portugal que avalia a perda auditiva em doentes com TB-MDR ou TB-XDR tratados com fármacos ototóxicos.

A perda auditiva constitui um dos efeitos mais devastadores, resultantes do uso de fármacos aminoglicosídeos nas formas resistentes de tuberculose. Acredita-se que se deve à apoptose das células ciliadas externas desencadeada pela formação de espécies reativas de oxigénio, ${ }^{2,9}$ o que poderá explicar a ausência de OEA, verificada neste estudo, em doentes com perda auditiva. A destruição das células ciliadas externas inicia-se na base da cóclea e progride para o ápex e gânglio espiral de Corti nas formas mais avançadas, o que faz com que a perda auditiva se manifeste, inicialmente, nas frequências elevadas. ${ }^{2,3,4,6}$ Este fenómeno é evidente no gráfico da Fig. 1, onde estão representadas as médias dos limiares auditivos em cada uma das frequências testadas. A falta de consenso na definição de perda auditiva e a elevada variabilidade nas metodologias de avaliação fazem com que os resultados disponíveis na literatura apresentem uma variabilidade entre $21,3 \%$ e $70,1 \%{ }^{2,3,5,7,10}$ No entanto, é interessante constatar que a proporção de doentes com perda auditiva é superior em estudos prospetivos, em que a avaliação foi estandardizada e alargada a todos os doentes, sugerindo que as formas clinicamente menos significativas de perda auditiva possam passar despercebidas em trabalhos de menores dimensões e com metodologias menos robustas.

Existem poucos fatores que, comprovadamente, aumentam a susceptibilidade para a ototoxicidade causada pelos aminoglicosídeos. A maioria dos fatores de risco

Tabela 1B - Dados da avaliação auditiva em doentes com TB-MDR tratados no CRRTB-MDR-Norte (fim).

\begin{tabular}{|c|c|c|c|c|c|c|c|c|}
\hline \multirow[t]{3}{*}{ Caso } & \multicolumn{8}{|c|}{ AVALIAÇÃO AUDITIVA } \\
\hline & \multirow{2}{*}{$\begin{array}{c}\text { Timpanograma } \\
\text { (tipo) }\end{array}$} & \multirow{2}{*}{ OEA } & \multirow{2}{*}{$\begin{array}{l}\text { PTA/SRT } \\
\text { (dB) }\end{array}$} & \multicolumn{4}{|c|}{ AT de altas frequências $(\mathrm{KHz})(\mathrm{dB})$} & \multirow[t]{2}{*}{ Otoscopi } \\
\hline & & & & 8 & 10 & 12.5 & 16 & \\
\hline \multirow{2}{*}{1} & A & Ausentes & $26 / 30$ & 65 & 60 & 80 & $\infty$ & \multirow{2}{*}{ Normal } \\
\hline & A & Ausentes & $33 / 20$ & 60 & 55 & 70 & $\infty$ & \\
\hline \multirow{2}{*}{2} & A & Ausentes & $100 / \infty$ & $\infty$ & $\infty$ & $\infty$ & $\infty$ & \multirow{2}{*}{ Normal } \\
\hline & A & Ausentes & $100 / \infty$ & $\infty$ & $\infty$ & $\infty$ & $\infty$ & \\
\hline \multirow{2}{*}{3} & $A$ & Ausentes & $65 / 55$ & 75 & 70 & $\infty$ & $\infty$ & \multirow{2}{*}{ Normal } \\
\hline & $A$ & Ausentes & $60 / 90$ & 90 & 80 & $\infty$ & $\infty$ & \\
\hline \multirow{2}{*}{4} & A & Ausentes & $62 / 55$ & 105 & 95 & $\infty$ & $\infty$ & \multirow[t]{2}{*}{ Normal } \\
\hline & A & Ausentes & $54 / 57$ & 85 & 70 & $\infty$ & $\infty$ & \\
\hline \multirow{2}{*}{5} & $A$ & Presentes & $14 / 37$ & 15 & 15 & 40 & $\infty$ & \multirow{2}{*}{ Normal } \\
\hline & A & Presentes & $12 / 27$ & 30 & 30 & 55 & $\infty$ & \\
\hline \multirow{2}{*}{6} & $A$ & Presentes & $23 / 37$ & 80 & 90 & $\infty$ & $\infty$ & \multirow{2}{*}{ Normal } \\
\hline & A & Presentes & $24 / 23$ & 70 & 75 & $\infty$ & $\infty$ & \\
\hline \multirow{2}{*}{7} & A & Ausentes & $53 / 50$ & 95 & $\infty$ & $\infty$ & $\infty$ & \multirow{2}{*}{ Normal } \\
\hline & $A$ & Ausentes & $58 / 50$ & 85 & $\infty$ & $\infty$ & $\infty$ & \\
\hline \multirow{2}{*}{8} & $A$ & Ausentes & $84 / \infty$ & 105 & $\infty$ & $\infty$ & $\infty$ & \multirow{2}{*}{ Normal } \\
\hline & $A$ & Ausentes & $80 / \infty$ & 100 & $\infty$ & $\infty$ & $\infty$ & \\
\hline \multirow{2}{*}{9} & A & Presentes & $17 / 27$ & 35 & 70 & 70 & $\infty$ & \multirow{2}{*}{ Normal } \\
\hline & $A$ & Ausentes & $35 / 33$ & 95 & 80 & $\infty$ & $\infty$ & \\
\hline \multirow{2}{*}{10} & A & Presentes & $10 / 20$ & 5 & 5 & 5 & 30 & \multirow{2}{*}{ Normal } \\
\hline & $A$ & Presentes & $9 / 15$ & 10 & 5 & 5 & 30 & \\
\hline
\end{tabular}




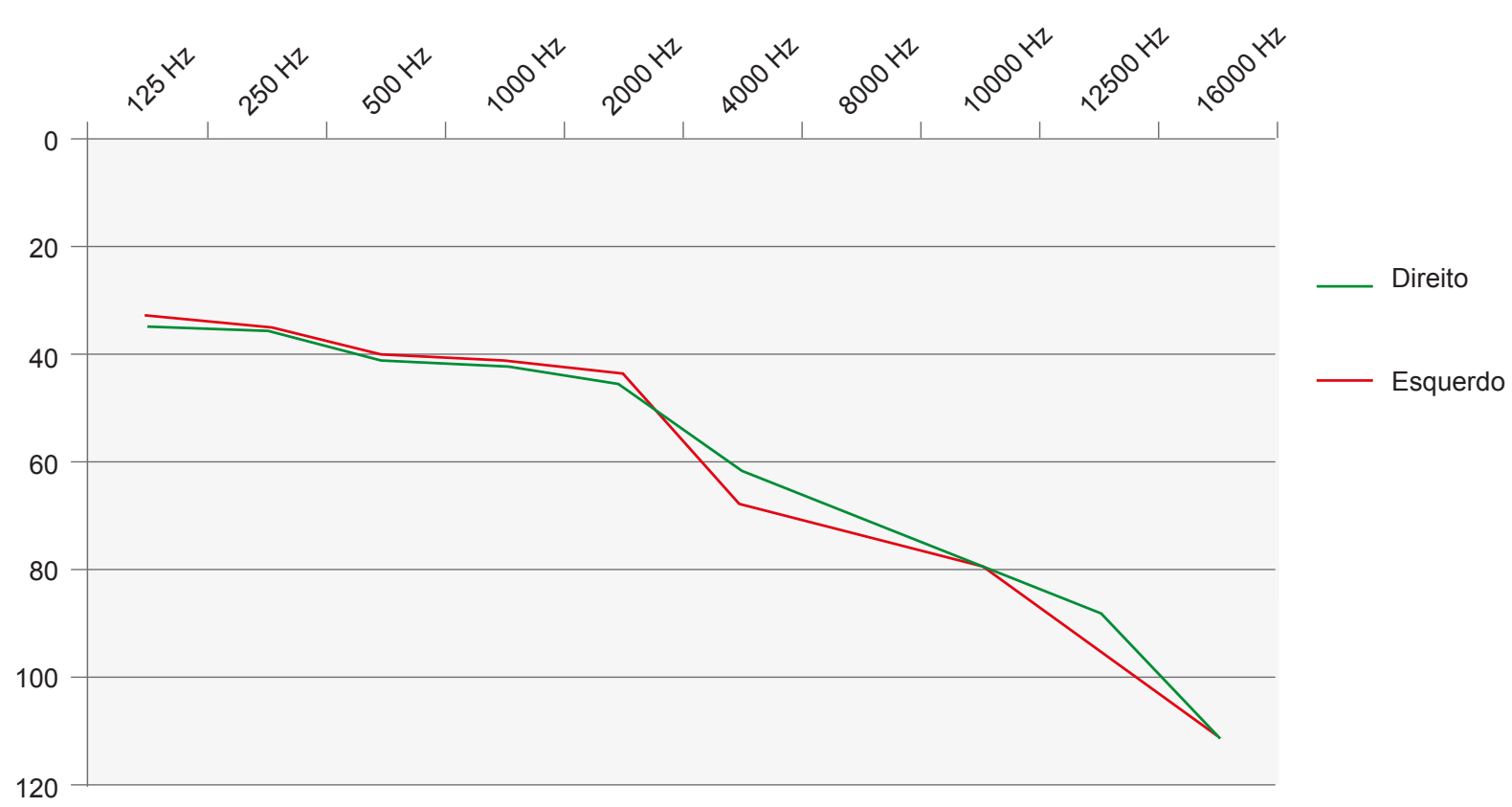

Figura 1 - Média dos limiares auditivos (em dB HL) nos indivíduos estudados em função da frequência

apontados em modelos animais são difíceis de comprovar ou não apresentam correlação em estudos humanos. Apesar de ter sido demonstrado, em estudos animais, que a ototoxicidade é mais prevalente com doses, frequências de administração e tempos de tratamento superiores, ${ }^{11}$ isto foi contrariado numa revisão sistemática de 48 estudos publicada em 2010.12 O mesmo acontece com outros fatores como a idade, sexo ou tabagismo. , $^{2,3,5,7} A$ predisposição genética é um fator de risco bem conhecido. A mutação A1555G no DNA mitocondrial está associada a surdez não sindrómica e aumenta a susceptibilidade à ototoxicidade provocada pelos aminoglicosídeos. ${ }^{13}$ Esta mutação poderá ser rastreada com a realização de testes genéticos, no futuro, de forma a excluir os doentes mais susceptíveis ao dano coclear ototóxico.

O fato de se tratar de um dano permanente e irreversível, faz com que a monitorização da função auditiva seja essencial durante o tratamento com fármacos ototóxicos. Apesar de não existir consenso entre as diferentes associações mundiais ${ }^{4}$, é aceite que a audição deve ser testada antes do início do tratamento, com um audiograma basal, e, depois, com uma frequência nunca inferior à mensal, até aos 3-6 meses após a sua descontinuação. 4,6,9,14 Essa avaliação deverá incluir as frequências superiores a 8000 $\mathrm{Hz}$, de forma a aumentar a sensibilidade do teste, uma vez que, como já abordado, a perda auditiva inicia-se e é mais acentuada nas frequências superiores. Qualquer perda de $20 \mathrm{~dB}$ numa frequência isolada ou de $10 \mathrm{~dB}$ em duas frequências contíguas deverá alertar o clínico para a ocorrência de ototoxicidade. ${ }^{14}$ Quando esta for detetada, as diferentes opções passam pela suspensão, redução da dose e frequência de administração, ou pela mudança do fármaco para outro menos ototóxico. A decisão a tomar deve ser individualizada e dependente da severidade da doença, do tempo em que o fármaco foi administrado, do perfil de sensibilidade da estirpe em causa, da disponibilidade de alternativas eficazes e seguras, bem como da severidade $e$ do tempo de instalação da perda auditiva.

O fato de se tratar de um estudo retrospetivo, sem uma avaliação auditiva de base ou pré-tratamento, faz com este estudo assuma uma limitação importante. Apesar de se ter excluído os sujeitos com queixas de perda auditiva antes da administração dos fármacos ototóxicos, isso não garante que nos indivíduos testados existissem formas de hipoacúsia subclínicas com implicações importantes na presente avaliação. Isso torna premente a realização de estudos prospetivos com um maior número de participantes e, talvez, de âmbito nacional, com a participação dos cinco Centros de Referência Regionais para a TB-MDR.

De igual forma, não podemos excluir a presença de viés de seleção, com a participação no estudo daqueles indivíduos com queixas de hipoacúsia.

\section{CONCLUSÃO}

A elevada incidência de perda auditiva detetada neste estudo, faz com que seja necessária a criação de protocolos de atuação entre os Centros de Referência e os Serviços de Otorrinolaringologia de proximidade, de forma a que esta seja precocemente detetada e orientada.

\section{OBSERVAÇÕES}

Trabalho apresentado como poster no $61^{\circ}$ Congresso da Sociedade Portuguesa de Otorrinolaringologia e Cirurgia Cérvico-Facial. Lisboa, 1-4 de Maio de 2014. 


\section{CONFLITOS DE INTERESSE}

Os Autores declararam a inexistência de qualquer conflito de interesses.

\section{REFERÊNCIAS}

1. Vilariça A, Gomes C, Pina J. Análise comparativa entre tuberculose multirresistente e tuberculose extensivamente resistente Epidemiologia e factores preditivos. Rev Por Pneumol. 2008;14:829-42.

2. Javadi MR, Abtahi B, Gholami K, Safari Moghadam B, Tabarsi P, Salamzadeh J. The incidence of amikacin ototoxicity in multidrugresistent tuberculosis patients. Iran J Pharm Res. 2011;10:905-11.

3. Seddon JA, Thee S, Jacobs K, Ebrahim A, Hesseling AC, Schaaf HS. Hearing loss in children treated for multidrug-resistent tuberculosis. J Infect. 2013;66:320-9.

4. Seddon JA, Godfrey-Faussett P, Jacobs K, Ebrahim A, Hesseling AC, Schaaf HS. Hearing loss in patients on treatment for drug-resistent tuberculosis. Eur Respir J. 2012;40:1277-86.

5. Ramma L, lbekwe T. Cochleo-vestibular clinical findings among drug resistant tuberculosis patients on therapy - a pilot study. Int Arch Med. 2012;31:5-13.

6. Duggal P, Sarkar M. Audiologic monitoring of multi-drug resistant tuberculosis patients on aminoglycoside treatment with long term followup. BMC Ear Nose Throat Disord. 2007;12:7-15.

7. Jager $P$, Altena R. Hearing loss and nephrotoxicity in long-term aminoglycoside treatment in patients with tuberculosis. Int $\mathrm{J}$ Tuberc Lung Dis. 2002;6:622-7.

8. Vasconcelos KA, Lima MA, Frota S, Ruffino Netto A, Kritski AL. Avaliação

\section{FONTES DE FINANCIAMENTO}

O presente trabalho não foi objecto de quaisquer financiamentos.

audiométrica de pacientes em tratamento para tuberculose pulmonar. J Bras Pneumol. 2012;38:81-7.

9. Xie J, Talaska A, Schacht J. New developments in aminoglycoside therapy and ototoxicity. Hear Res. 2011;281: 28-37.

10. Törün T, Güngör $G$, Ozmen I, Bölükbaşi $Y$, Maden E, Biçakçi B, et al. Side effects associated with the treatment of multidrug resistant tuberculosis. Int J Tuberc Lung Dis. 2005:9:1373-7.

11. Wu WJ, Sha SH, McLaren JD, Kawamoto K, Raphael Y, Schacht J. Aminoglycoside ototoxicity inadult CBA, C57BL and BALB mice and the Sprague-Dawley rat. Hear Res. 2001;158:165-78.

12. America Speech-Language-Hearing Association. Evidence-based systematic review (ESBR): drug-induced hearing loss - aminoglycosides. 2010. [Consultado 2014 Fev 03]; Disponível em http://www.asha.org/.

13. Prezant TR, Agapian JV, Bohlman MC, Bu X, Oztas S, Qiu WQ, et al. Mitochondrial ribosomal RNA mutation associated with both antibioticinduced and non-syndromic deafness. Nat Genet. 1993;4:289-94.

14. America Speech-Language-Hearing Association. Audiological Management of Individuals Receiving Cochleotoxic Drug Therapy (Guideline). Rockeville, America Speech-Language-Hearing Association, 1994. [Consultado 2014 Fev 03]. Disponível em http://www. asha.org/. 
Leandro RIBEIRO, Claudia SOUSA, Aida SOUSA, Catarina FERREIRA, Raquel DUARTE, António FARIA E ALMEIDA, Artur CONDÉ

\section{Avaliação da Perda Auditiva em Doentes com Tuberculose Multirresistente}

Acta Med Port 2015:28:87-91

Publicado pela Acta Médica Portuguesa, a Revista Científica da Ordem dos Médicos

Av. Almirante Gago Coutinho, 151

1749-084 Lisboa, Portugal.

Tel: +351218428 215

E-mail: submissao@actamedicaportuguesa.com

www.actamedicaportuguesa.com

ISSN:0870-399X | e-ISSN: 1646-0758

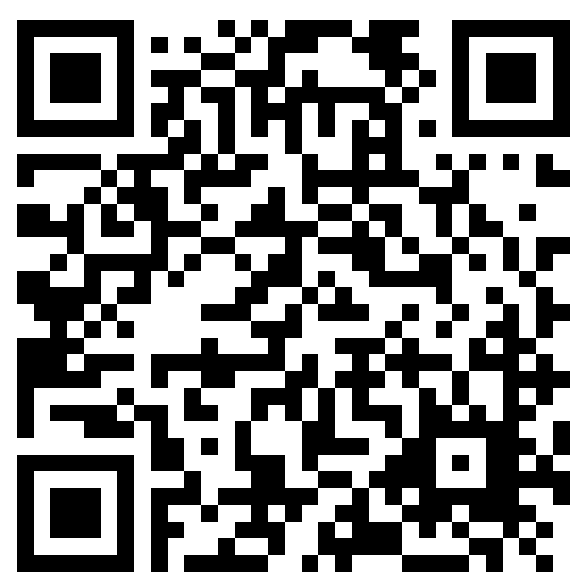

